\title{
Antioxidants linked with physical, cognitive and psychological frailty: Analysis of candidate biomarkers and markers derived from the MARK-AGE study
}

\author{
M. Liset Rietman ${ }^{\mathrm{a}, \mathrm{b}, *}$, Annemieke M.W. Spijkerman ${ }^{\mathrm{a}}$, Albert Wong ${ }^{\mathrm{a}}$, Harry van Steeg ${ }^{\mathrm{a}}$, \\ Alexander Bürkle $^{\mathrm{c}}$, María Moreno-Villanueva ${ }^{\mathrm{c}}$, Thilo Sindlinger ${ }^{\mathrm{c}}$, Claudio Franceschi ${ }^{\mathrm{d}}$, \\ Beatrix Grubeck-Loebenstein ${ }^{\mathrm{e}}$, Jürgen Bernhardt ${ }^{\mathrm{f}}$, P. Eline Slagboom ${ }^{g}$, Olivier Toussaint ${ }^{\mathrm{h}, 1}$, \\ Florence Debacq-Chainiaux ${ }^{\mathrm{h}}$, Ewa Sikora ${ }^{\mathrm{i}}$, Efstathios S. Gonos ${ }^{\mathrm{j}}$, Nicolle Breusing ${ }^{\mathrm{k}}$, \\ Wolfgang Stuetz ${ }^{\mathrm{p}}$, Daniela Weber ${ }^{1}$, Tilman Grune ${ }^{1}$, Andrea Basso ${ }^{\mathrm{m}}$, Francesco Piacenza ${ }^{\mathrm{m}}$, \\ Marco Malavolta $^{\mathrm{m}}$, Sebastiano Collino ${ }^{\mathrm{n}}$, Eugene H.J.M. Jansen ${ }^{\mathrm{a}}$, W. M. Monique Verschuren ${ }^{\mathrm{a}, \mathrm{b}}$, \\ Martijn E.T. Dollé,o
}

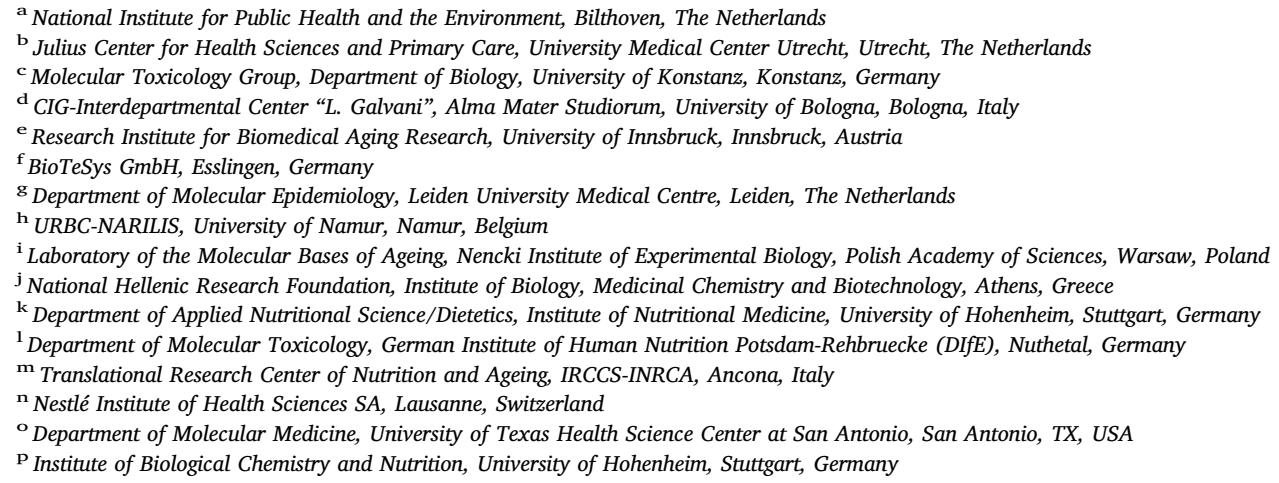

Keywords:

Frailty

Ageing

Multidimensional

Univariate

Multivariate

Machine learning

Elastic net

\begin{abstract}
Frailty among elderly people leads to an increased risk for negative health outcomes. To prevent frailty, we need a better understanding of the underlying mechanisms and early detection of individuals at risk. Both may be served by identifying candidate (bio)markers, i.e. biomarkers and markers, for the physical, cognitive, and psychological frailty domains. We used univariate (Rank-ANOVA) and multivariate (elastic net) approaches on the RASIG study population (age range: 35-74 years, $\mathrm{n}=2220$ ) of the MARK-AGE study to study up to 331 (bio)markers between individuals with and without frailty for each domain. Biomarkers and markers identified by both approaches were studied further regarding their association with frailty using logistic regression. Univariately, we found lower levels of antioxidants, including $\beta$-cryptoxanthin and zeaxanthin, in those who were physically, cognitively or psychologically frail. Additionally, self-reported health was worse in these three frail groups. Multivariately, we observed lower levels of $\beta$ cryptoxanthin and zeaxanthin in the cognitively frail. Levels of these carotenoids were inversely associated with the risk of being cognitively frail after adjusting for confounders. Antioxidants and self-reported health are potential (bio)markers to detect persons at risk of becoming frail. The biomarkers identified may indicate the involvement of inflammation in frailty, especially for physical and cognitive frailty.
\end{abstract}

\footnotetext{
* Corresponding author at: National Institute for Public Health and the Environment, Centre for Nutrition, Prevention and Health Services, PO Box 1, 3720 BA, Bilthoven, The Netherlands.

E-mail address: liset.rietman@rivm.nl (M.L. Rietman).

${ }^{1}$ Deceased.
} 


\section{Introduction}

Frailty is an age-associated syndrome and can be described as the accumulation of deficits in physical, cognitive, psychological or social functioning, leading to an increased risk for negative health outcomes (Walston et al., 2006) such as injuries including fractures through falls, disability, hospitalization, institutionalization, and mortality (Fried et al., 2001; Rockwood et al., 1999; Speechley and Tinetti, 1991; Winograd et al., 1991). To prevent or if possible reverse frailty, we need a better understanding of the underlying mechanisms of frailty. Identifying biomarkers could contribute to our knowledge about these mechanisms. In addition, the mechanisms behind frailty could help target preventive interventions. Furthermore, (bio)markers, i.e. biomarkers and markers, could be a valuable tool for early detection of those at increased risk of frailty. Evidence is accumulating that chronic low-grade inflammation may be involved in the development of frailty (Hamerman, 1999; Leng et al., 2007; Morley and Baumgartner, 2004; Walston et al., 2002). Hubbard et al. (2009) found that several inflammatory biomarkers such as C-reactive protein (CRP) and interleukin-6 (IL-6) were associated with two frailty definitions. Besides inflammation and the related oxidative stress process, several other biological processes have been linked to frailty such as hematological processes, immunosenescence, and other forms of cellular aging (Collerton et al., 2012; Mitnitski et al., 2015; Saum et al., 2015).

Over the years, many frailty instruments have been developed with the focus on 'physical frailty', or the 'accumulation of deficits', or a 'multidimensional' approach to frailty with little agreement between instruments (Aguayo et al., 2017). With respect to biomarker research, most studies examined biomarkers for physical frailty, or for the accumulation of deficits. Only few studied associations between biomarkers and specific frailty domains, such as the association between brain-derived neurotrophic factor (BDNF) and cognitive frailty (Ingles et al., 2016). Our aim is to identify (bio)markers for three specific frailty domains, i.e. the physical, cognitive and psychological domains. The rationale of this approach is based on our previous study showing limited overlap between different frailty domains suggestive of distinct sub-groups (Rietman et al., 2018). Moreover, this approach may provide additional evidence on whether the underlying mechanisms per domain differ or not.

\section{Methods}

\subsection{Participants and study design}

The 'European study to establish bioMARKers of human AGEing' (MARK-AGE) is a consortium aiming to study biomarkers of human ageing. The MARK-AGE study, as previously described (Burkle et al., 2015), is a cross-sectional study and includes multiple populations. For the present analyses we used the 'Randomly recruited Age-Stratified Individuals from the General population' (RASIG) study population within the age-range of 35-74 years $(n=2220)$ in accordance to the MARK-AGE study design. We excluded 2 participants with a body mass index $(\mathrm{BMI})>55 \mathrm{~kg} / \mathrm{m}^{2}$ and we excluded the Finnish participants $(n=70)$ because this is a population isolate (Peltonen et al., 1999). There were 184 missing observations for the cognition data. As a result, the analyses for physical and psychological frailty each included 2128 participants, whilst the analysis for cognitive frailty comprised 1944 participants.

\subsection{Measurements}

Below we will briefly describe the different measurements that are relevant for the present analyses. The standard operating procedures, including collection of biological material, have been previously described (Moreno-Villanueva et al., 2015a).

Upon written informed consent the following information was obtained with a standardized questionnaire: sociodemographic factors (sex, age, level of education, marital status), lifestyle factors (smoking status, nutrition, physical activity), health status (self-reported health, falls, hospitalization), mood (ZUNG depression scale), and mental health (Mental Health Inventory-5). Weight and height were measured to calculate the BMI. Further, waist and hip circumference, blood pressure, heart rate at rest, and handgrip strength were measured. Immediate and delayed memory function was tested using the 15Picture Word Learning test (Brand and Jolles, 1985). Cognitive flexibility was measured with the Stroop test (Stroop, 1935) and cognitive speed was tested using the Digit Symbol Substitution test (Lezak et al., 2004). Using the biological material (plasma, serum, peripheral blood mononuclear cells, and urine), an extensive set of biomarkers was measured such as DNA-based, immunological, and oxidative stress biomarkers. The complete list of biomarkers has been described earlier (Moreno-Villanueva et al., 2015b).

\subsection{Frailty criteria}

We used a multidimensional approach to study frailty. Physical frailty was defined according to the Frailty Phenotype (Fried et al., 2001). Participants were considered physically frail if they fulfilled at least 2 out of 4 frailty criteria described by Fried: unintentional weight loss, exhaustion, low physical activity and reduced handgrip strength (Supplementary material: Appendix A). Because gait speed was not available, we used 4 instead of 5 criteria for physical frailty and we adapted the cut-off point for physical frailty accordingly.

The other frailty domains were based on the Tilburg Frailty Indicator (Gobbens et al., 2010). Our operationalization of these domains has been previously described (Rietman et al., 2018).

Participants were considered cognitively frail when scoring below the 10th percentile on global cognitive functioning. Global cognitive functioning was based on the scores of participants on the 15-Picture Word Learning test, the Stroop test, and Digit Symbol Substitution test. These scores were transformed into z-scores and combined into one global cognitive functioning score that was adjusted for level of education.

Psychological frailty was defined as fulfilling 2 out of 2 criteria: depressive symptoms and poor mental health. Depressive symptoms were assessed with 20 questions of the Zung self-rating depression scale (ZUNG) (Zung, 1965). Those with a score of $>50$ (out of a range of 0-100) were considered to have depressive symptoms. Mental health status was measured with the Mental Health Inventory-5 (Berwick et al., 1991). Scores of five questions on a six-point scale were transformed into a total score ranging from 0 to 100 . A score of 60 or below indicated reduced mental health (Perenboom et al., 2000).

\section{Statistical analyses}

The MARK-AGE dataset as of November 2015 was used for the statistical analyses. Prior to the analyses we made a pre-selection by removing (bio)markers that were measured only in a fraction of the cohort, or that were unlikely to be related to one of the frailty domains (mainly questionnaire data), or for which batch corrected data was available resulting in 
a list with 371 (bio)markers (Supplementary material: Appendix B; including details on missing observations per frailty domain).

Descriptive analyses were carried out for the non-frail, physically frail, cognitively frail, and psychologically frail groups separately.

Following Saccenti et al. (2014), we performed two complementary analyses, univariate and multivariate analyses, to identify candidate (bio)markers for three frailty domains, both having their advantages and disadvantages (Supplementary material: Appendix C). With the univariate analyses, we tested the null hypothesis that the frail group had the same (bio)marker level as the non-frail group. This was tested for each frailty domain and each (bio)marker using Rank-ANOVA, a non-parametric version of ANOVA, while adjusting for sex and age. Since Rank-ANOVA does not accept all character variables, 331 of the 371 (bio)markers were studied with this approach. To control for false discoveries, we used the Benjamini-Hochberg correction for multiple testing (Benjamini and Hochberg, 1995). Because of biological differences between men and women, analyses were repeated in a sensitivity analysis where we stratified for sex. The analyses were carried out in SAS 9.3 and SAS 9.4 for Windows (SAS Institute Inc., Cary, NC, USA).

Our multivariate approach was based on machine learning techniques. We used a variable selection procedure based on Stability Selection (Meinshausen and Bühlmann, 2010) in combination with elastic net (Zou and Hastie, 2005) to identify candidate (bio)markers. Since this procedure can only be applied on observations without missing values (most (bio)markers were not measured for all individuals), we trimmed the candidate (bio)markers-list in such a way as to maximize the sum of the fraction of (bio)markers (i.e. covariates) and the fraction of frail individuals. This resulted in 55 frail cases and 222 (bio)markers for physical frailty, 141 frail cases and 275 (bio)markers for cognitive frailty, and 117 frail cases and 219 (bio)markers for psychological frailty. Next, skewed (bio)markers were transformed using the Yeo-Johnson power transformation (Yeo and Johnson, 2000). Since our dataset was imbalanced (i.e. low number of frail cases and high number of non-frail controls) and we needed to adjust for sex and age, we used a form of undersampling (Blagus and Lusa, 2015; Wallace et al., 2011) prior to the Stability Selection procedure. In our approach, we sampled controls in such a way that within each age and sex stratum (age in five-year categories) the number of cases and controls were identical. As undersampling leads to extra sampling variability, we repeated our approach 10,000 times. In each iteration, undersampling was used to create a balanced dataset, the variable selection procedure was applied, and a list of variable selection probabilities was obtained. A final ranking of variable selection probabilities was obtained by averaging the selection probabilities over all iterations. Biomarkers or markers with an average selection probability of $>0.5$ were used for further analyses, in which the risk of being frail was calculated by subtracting the average value of the 10th percentile for a certain (bio)marker from the 90th percentile from that (bio)marker, i.e. the delta. Given the low number of cases, a sensitivity analyses with stratification for sex was not possible for the multivariate analyses. The multivariate approach was carried out in R 3.3.2.

Biomarkers or markers identified by both approaches were studied as covariates in a binomial logistic regression model to obtain effect sizes of each (bio)marker. There we adjusted for confounders based on literature (Michelon et al., 2006) being sex, age, level of education, BMI, smoking status and season of blood collection. For cognitive frailty, we adjusted for depressive symptoms in addition. Technical details on the statistical analysis can be found in Appendix $C$ of the Supplementary material.

\section{Results}

\subsection{Population characteristics}

Characteristics of the groups with and without frailty stratified by the different frailty domains are presented in the baseline table
(Table 1). The psychologically frail group was younger than the physically and cognitively frail groups. Psychological frailty was more common among women, while cognitive frailty was more common among men. The cognitively frail group had a higher systolic and diastolic blood pressure than the physically and psychologically frail groups. All three frail groups had a lower educational level and higher BMI than the nonfrail group. In addition, the physically, cognitively, and psychologically frail groups showed limited overlap (Fig. 1).

\subsection{Prevalence}

A total of 64 (3.0\%) participants were physically frail, 199 (10.2\%) were (by definition) cognitively frail, and 134 (6.3\%) were psychologically frail (Fig. 1). When studying the prevalence in five-year age groups per frailty domain, the prevalence of physical and cognitive frailty rapidly increased from age 60 onwards, while the prevalence of psychological frailty did not show a pronounced age-related increase (Fig. 2a). Stratified by sex, Fig. $2 \mathrm{~b}$ confirms the sex-specific prevalence for $p s y$ chological and cognitive frailty across all age groups. Furthermore, it shows similar slopes for men and women within frailty domains.

\subsection{Candidate (bio)markers for different frailty domains: results from univariate analyses}

All three frail groups reported to have worse health compared to the non-frail groups (Table 2). In physically frail people, significantly lower levels of $\alpha$-carotene, selenium (total and sub-fraction), $\beta$-cryptoxanthin, and $\beta$-carotene were observed compared to people without physical frailty, while higher monocyte levels were observed. In cognitively frail people, levels of $\beta$-cryptoxanthin, zeaxanthin, cholesterol, $\alpha$-tocopherol, and guanidinoacetate were significantly lower compared to people without cognitive frailty, while cytomegalovirus (CMV) antibody levels were significantly higher. In psychologically frail people, levels of $\alpha$-carotene were significantly lower compared to people without psychological frailty, and also level of education was statistically significantly lower (Table 2). Age stratified boxplots of the biomarkers listed in Table 2, visualize the spread in expression of each biomarker and clarify the level of overlap in expression when comparing the frail and non-frail groups (Supplementary material: Appendix D).

\subsection{Sensitivity analyses: results from univariate analyses with stratification for sex}

In physically frail women, levels of $\alpha$-carotene and $\beta$-carotene were significantly lower compared to women without physical frailty. In psychologically frail women, level of education was significantly lower compared to women without psychological frailty (Table 3a).

In cognitively frail men, levels of $\beta$-cryptoxanthin and guanidinoacetate were significantly lower, while levels of CMV antibodies were significantly higher compared to men without cognitive frailty. In psychologically frail men, levels of copper not bound to ceruloplasmin were significantly lower compared to men without psychological frailty (Table 3b).

Both men and women in all three frail groups reported to have worse health compared to the non-frail groups (Tables 3a and 3b). Age and sex stratified boxplots of the biomarkers listed in Tables 3a and 3b, visualize the spread in expression of each biomarker and clarify the level of overlap in expression when comparing the frail and non-frail groups (Supplementary material: Appendix D).

\subsection{Candidate (bio)markers for different frailty domains: results from multivariate analyses}

The multivariate approach did not lead to a selection of (bio)markers that were predictive of physical and psychological frailty, 
Table 1

Characteristics table of the non-frail and the frail groups.

\begin{tabular}{|c|c|c|c|c|}
\hline Variables & $\begin{array}{l}\text { Non-frail }^{\text {a }} \\
N=1628\end{array}$ & $\begin{array}{l}\text { Physically frail } \\
N=64\end{array}$ & $\begin{array}{l}\text { Cognitively frail } \\
\mathrm{N}=199\end{array}$ & $\begin{array}{l}\text { Psychologically frail } \\
\mathrm{N}=134\end{array}$ \\
\hline \multicolumn{5}{|l|}{ Socio-demographic factors } \\
\hline Women, \% (n) & $51.4(837)$ & $50.0(32)$ & $37.7(75)$ & $70.9(95)$ \\
\hline Age (years), mean (SD), & $53.4(11.0)$ & $62.8(10.6)$ & $64.3(8.5)$ & $57.8(10.5)$ \\
\hline Low level of education, \% (n) & $8.7(141)$ & $20.6(13)$ & $11.6(23)$ & $24.6(33)$ \\
\hline Marital status widowhood, \% (n) & $5.0(82)$ & $10.9(7)$ & $11.1(22)$ & $11.2(15)$ \\
\hline \multicolumn{5}{|l|}{ Anthropometric data } \\
\hline $\mathrm{BMI}^{\mathrm{b}}$, mean $(\mathrm{SD})$ & $25.7(4.2)$ & $28.4(5.3)$ & $27.5(4.3)$ & $26.8(4.7)$ \\
\hline Waist circumference, mean (SD) & $90.7(13.8)$ & $94.8(12.4)$ & $96.0(11.3)$ & $91.9(13.6)$ \\
\hline Systolic blood pressure, mean (SD) & $132.7(19.0)$ & $138.5(21.8)$ & $142.2(20.3)$ & $130.7(22.2)$ \\
\hline Diastolic blood pressure, mean (SD) & $80.7(10.7)$ & $82.4(11.3)$ & $84.5(12.4)$ & $78.8(11.0)$ \\
\hline \multicolumn{5}{|l|}{ Life-style factors } \\
\hline Low physical activity ${ }^{\mathrm{c}}, \%$ (n) & $10.3(54)$ & $58.0(29)$ & $19.0(29)$ & $20.0(12)$ \\
\hline Current smoker, \% (n) & $17.6(287)$ & $25.0(16)$ & $16.6(33)$ & $26.1(35)$ \\
\hline Low fruit consumption ${ }^{\mathrm{d}}, \%(\mathrm{n})$ & $21.5(350)$ & $20.3(13)$ & $17.6(35)$ & $32.8(44)$ \\
\hline Low vegetable consumption ${ }^{\mathrm{d}}, \%(\mathrm{n})$ & $16.9(275)$ & 21.9 (14) & $13.1(26)$ & $21.6(29)$ \\
\hline Low vitamin supplement use ${ }^{\mathrm{d}}, \%$ (n) & $85.6(1394)$ & $81.3(52)$ & $81.4(162)$ & $85.1(114)$ \\
\hline \multicolumn{5}{|l|}{ Health and disease } \\
\hline Poor/fair self-reported health, \% (n) & $5.7(93)$ & $51.6(33)$ & $27.1(54)$ & $44.8(60)$ \\
\hline Falls ${ }^{\mathrm{e}}, \%(\mathrm{n})$ & $8.4(137)$ & $12.5(8)$ & $10.6(21)$ & $19.4(26)$ \\
\hline Hospitalisation $^{\mathrm{f}}, \%$ (n) & $11.0(179)$ & $31.3(20)$ & $13.1(26)$ & $16.4(22)$ \\
\hline \multicolumn{5}{|l|}{ Season of blood collection } \\
\hline Winter, \% (n) & $19.4(314)$ & $15.6(10)$ & $24.1(48)$ & $18.7(25)$ \\
\hline Spring, \% (n) & $34.6(561)$ & $37.5(24)$ & $35.2(70)$ & $40.3(54)$ \\
\hline Summer, \% (n) & $24.0(389)$ & $21.9(14)$ & $14.1(28)$ & $20.9(28)$ \\
\hline Fall, \% (n) & $22.0(357)$ & $25.0(16)$ & $26.6(53)$ & $20.2(27)$ \\
\hline \multicolumn{5}{|l|}{ Biomarkers $^{\mathrm{g}}$} \\
\hline$\alpha$-Carotene $(\mu \mathrm{mol} / \mathrm{l})$, median (IQR) & $0.15(0.09-0.25)$ & $0.10(0.05-0.16)$ & $0.12(0.07-0.21)$ & $0.12(0.07-0.19)$ \\
\hline$\beta$-Carotene $(\mu \mathrm{mol} / \mathrm{l})$, median (IQR) & $0.58(0.37-0.88)$ & $0.39(0.26-0.55)$ & $0.48(0.30-0.77)$ & $0.52(0.30-0.84)$ \\
\hline$\beta$-Cryptoxanthin $(\mu \mathrm{mol} / \mathrm{l})$, median (IQR) & $0.22(0.12-0.38)$ & $0.15(0.07-0.29)$ & $0.15(0.07-0.37)$ & $0.19(0.10-0.38)$ \\
\hline Zeaxanthin $(\mu \mathrm{mol} / \mathrm{l})$, median (IQR) & $0.044(0.028-0.065)$ & $0.040(0.023-0.063)$ & $0.036(0.022-0.054)$ & $0.039(0.025-0.058)$ \\
\hline$\alpha$-Tocopherol ( $\mu \mathrm{mol} / \mathrm{l})$, median (IQR) & $27.8(23.6-32.6)$ & $27.6(23.0-33.7)$ & $26.6(22.5-32.1)$ & $27.6(23.3-32.4)$ \\
\hline Selenium (ppb), median (IQR) & $110(97-124)$ & $99(88-113)$ & $107(92-121)$ & $108(97-119)$ \\
\hline SeAlbSeP $(\mathrm{ppb})^{\mathrm{h}}$, median (IQR) & $88(76-99)$ & $78(68-89)$ & $85(71-98)$ & $86(76-95)$ \\
\hline CunotCp (ppb) ${ }^{\mathrm{i}}$, median (IQR) & $169(146-195)$ & 167 (151-192) & $161(143-188)$ & $170(137-201)$ \\
\hline Monocytes $\left(10^{6} / \mathrm{l}\right)$, median (IQR) & $442(360-554)$ & $526(450-640)$ & $499(420-615)$ & $470(382-592)$ \\
\hline CMV $(\mathrm{U} / 1)^{\mathrm{j}}$, median $(\mathrm{IQR})$ & $19.1(1.8-60.2)$ & $50.6(4.7-100.2)$ & $36.5(9.9-96.7)$ & $36.3(3.4-82.4)$ \\
\hline Guanidinoacetate $3.97 \mathrm{~s}$ (area (a.u.)) $)^{\mathrm{k}}$, median (IQR) & $8.4(5.7-12.2)$ & $7.1(4.7-11.3)$ & $7.6(5.3-11.5)$ & $8.4(5.7-12.4)$ \\
\hline Cholesterol (mmol/l), median (IQR) & $5.5(4.9-6.2)$ & $5.3(4.6-6.0)$ & $5.3(4.7-6.0)$ & $5.5(4.8-6.0)$ \\
\hline
\end{tabular}

a Participants in the non-frail group were not frail for any frailty domain.

b BMI: Body mass index $\left(\mathrm{kg} / \mathrm{m}^{2}\right)$.

c Physical activity was not measured in participants $<60$ yrs. We defined participants $<60$ years as being physically active. Participants were considered low physically active if they did not participate in light housework or exercise every day, or almost every day.

${ }^{d}$ Low fruit consumption, low vegetable consumption, and low vitamin supplement use were defined by combining the consumption frequencies of ' $1-3$ times a week', '1-3 times a month', and 'never'.

e Falls was defined as one or more falls in the last 12 months.

${ }^{\mathrm{f}}$ Hospitalization was defined as being hospitalized (with an overnight stay) within the last 12 months.

g Selected biomarkers based on findings of our subsequent analyses; the median and interquartile range (IQR) of untransformed biomarkers.

${ }^{\mathrm{h}}$ SeAlbSeP (ppb): Plasma Selenium eluting with retention time of Albumin or Selenoprotein P (absolute value in ppb).

i CunotCp (ppb): Plasma Copper not bound to Ceruloplasmin (absolute value in ppb).

j CMV: Cytomegalovirus antibodies (U/1).

k Relative Guanidinoacetate 3.97 s concentration.

based on an average selection probability $>0.5$ (Table 4). However, for cognitive frailty an average selection probability $>0.5$ was obtained for $\beta$-cryptoxanthin and zeaxanthin (Table 4). People with high levels (90th percentile) of $\beta$-cryptoxanthin had a $9.3 \%$ lower risk of being cognitively frail compared to people with low levels (10th percentile) of $\beta$-cryptoxanthin. People with high levels of zeaxanthin had $6.3 \%$ lower risk of being cognitively frail compared to people with low levels of zeaxanthin (Table 5).

\subsection{Associations between candidate biomarkers and cognitive frailty adjusted for confounders}

Based on both the univariate and multivariate analyses, we identified
B-cryptoxanthin and zeaxanthin as candidate biomarkers for cognitive frailty. With binomial logistic regression, we studied the association between these log-transformed carotenoids and cognitive frailty. In model 1, we adjusted for sex and age. In model 2, we adjusted for sex, age, level of education, BMI, smoking status, depressive symptoms and season of blood collection. In model $1, \log \beta$-cryptoxanthin (odds ratio 0.642 ; 95\% CI, 0.538-0.765) $\mathrm{p}<0.0001$ and $\log$ zeaxanthin (odds ratio 0.626; 95\% CI, 0.509-0.771) p < 0.0001 were associated with a lower risk of being cognitively frail. Also in model $2, \log \beta$-cryptoxanthin (odds ratio $0.742 ; 95 \% \mathrm{CI}, 0.604-0.911$ ) $\mathrm{p}=0.0043$ and $\log$ zeaxanthin (odds ratio $0.752 ; 95 \% \mathrm{CI}, 0.588-0.960) \mathrm{p}=0.0225$ were associated with a lower risk of being cognitively frail (Table 6). 


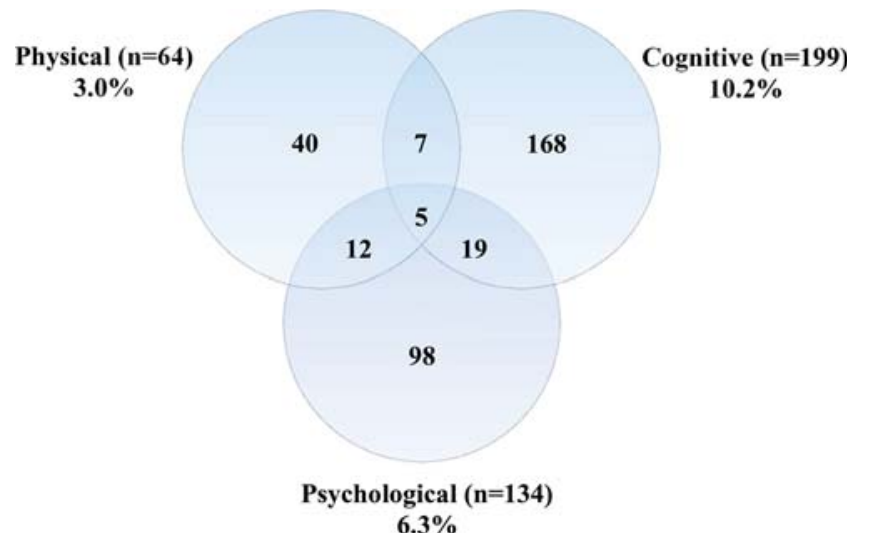

Fig. 1. Venn diagram showing the prevalence and overlapping individuals between the different frailty domains (i.e. the physically, cognitively and psychologically frail groups).

\section{Discussion}

We showed that women are more frequently psychologically frail, while men are more frequently cognitively frail. The prevalence of both physical and cognitive frailty increased with age, whereas the prevalence of psychological frailty hardly did (Fig. 2). Hence, the physical and cognitive frailty domains seem to be age-related, while psychological frailty
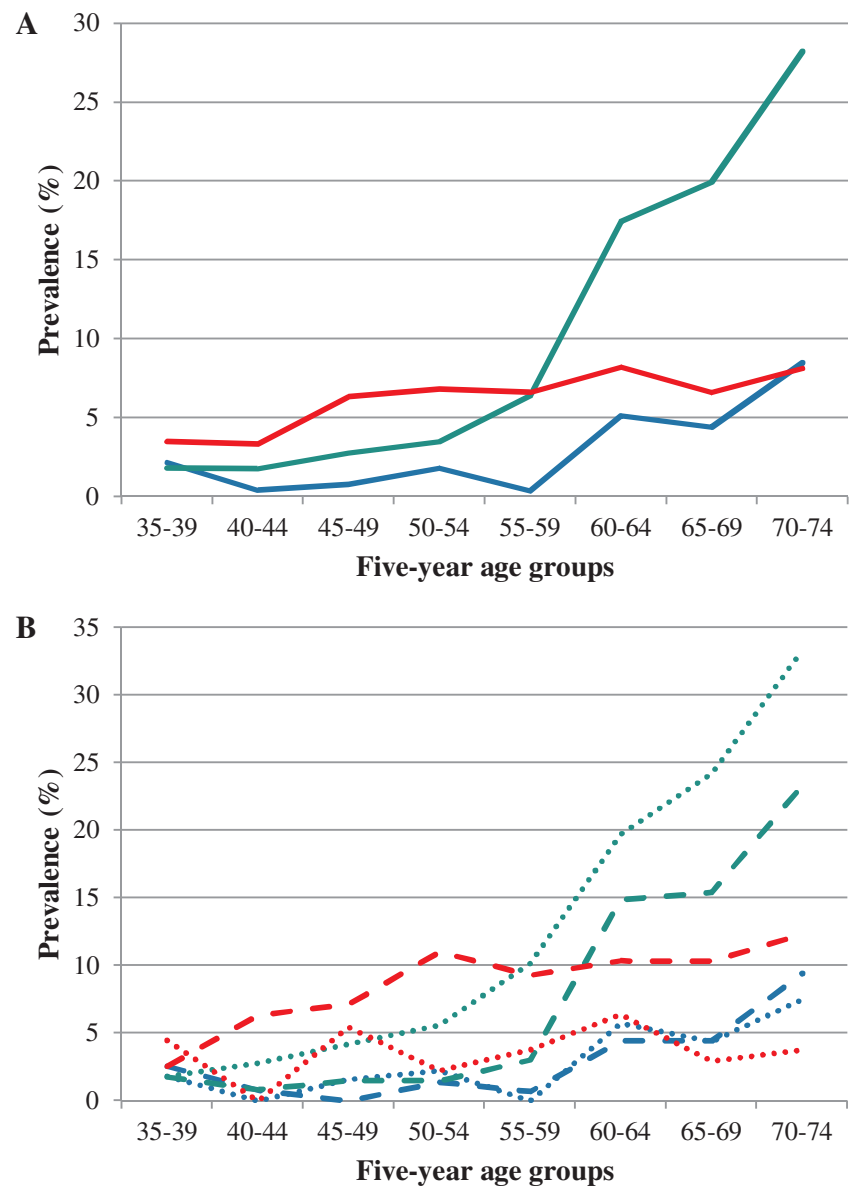

Fig. 2. Prevalence in five-year age groups per frailty domain for the total population (A) and stratified by sex (B). Blue line: physical frailty; green line: cognitive frailty; red line: psychological frailty; straight line: total population; striped line: women; dotted line: men. does not seem to be age-related. These findings are in line with our previous study in a different cohort, i.e. the Doetinchem Cohort Study (Rietman et al., 2018). In addition, both independent cohorts, the MARK-AGE study (Fig. 1) and the Doetinchem Cohort Study (Rietman et al., 2018), showed limited overlap between the frailty domains. Therefore, we identified candidate biomarkers for each frailty domain independently in the MARK-AGE study. Two complementary approaches, i.e. univariate and multivariate analyses, were applied from which three types of antioxidants (i.e. carotenoids, metals, tocopherol) emerged for one or more frailty domains.

Carotenoids were identified for all three domains. Univariately, we found lower levels of carotenoids in physically frail people compared to people without physical frailty when correcting for sex. When stratifying for sex we found lower levels of carotenoids in physically frail women. Other cross-sectional studies also found lower carotenoid levels in physically frail men and women (Smit et al., 2013) and in physically frail women (Michelon et al., 2006) compared to people without physical frailty. Both univariately and multivariately, we found lower levels of two carotenoids, i.e. $\beta$-cryptoxanthin and zeaxanthin, in cognitively frail people. This is in line with Dominguez and Barbagallo (2017), they suggested the involvement of antioxidants in cognitive frailty. Moreover, studies do report associations between carotenoids, such as zeaxanthin, and cognitive functioning (Akbaraly et al., 2007; Feeney et al., 2017). When stratifying for sex in the univariate analyses, we found lower levels of $\beta$-cryptoxanthin for cognitively frail men, but not for women. When studying the association between these carotenoids and cognitive frailty adjusted for important confounders, we showed that having higher levels of carotenoids was significantly associated with a lower risk of being cognitively frail. Univariately, we found lower levels of $\alpha$-carotene in psychologically frail people. Black et al. (2016) found that depressive symptoms were cross-sectionally associated with decreased carotenoid levels including $\alpha$-carotene. The percentage of people with and without frailty in our study population with low fruit, vegetable, or vitamin supplement consumption does not seem to differ considerably (Table 1). However, previously we showed that several of these carotenoids were associated with age of which a subset remained significant after adjusting for important confounders including dietary habits (Stuetz et al., 2016). Hence, the difference in carotenoid levels between frail and non-frail people might be caused by differences in nutrient intake but also by differences in nutrient absorption in the digestive system or in micronutrient metabolism with variation between carotenoids.

Similar to carotenoids, metals may also serve as pro-oxidants (Valko et al., 2006; Vina et al., 2016). We found lower levels of selenium in physically frail people, which has been reported before (Smit et al., 2013). We also found lower levels of copper (not eluting in the ceruloplasmin region) in psychologically frail men. It has been suggested that a mineral deficiency might trigger low-grade chronic inflammation (Maggio et al., 2013).

We identified one type of tocopherol, i.e. $\alpha$-tocopherol (a form of vitamin E), as a biomarker for cognitive frailty. This is in line with the suggested involvement of antioxidants in cognitive frailty (Dominguez and Barbagallo, 2017).

Antioxidants are, to a greater or lesser extent, associated with all frailty domains. Antioxidants could minimize the damaging effect of pro-inflammatory compounds such as inflammatory cytokines, like IL-6 and TNF- $\alpha$. The production of cytokines is upregulated via the activation of redox-sensitive transcription factors like nuclear factor kappa B (NF-kB) in response to a high level of reactive oxygen species (ROS) which causes oxidative stress (Semba et al., 2007). Indeed, it has previously been hypothesized that both inflammation and oxidative stress are associated with frailty (Hamerman, 1999; Maggio et al., 2006). Some of the biomarkers we identified are related to inflammatory processes such as monocytes, which we identified as a biomarker for physical frailty, and CMV, which we identified as a biomarker for cognitive frailty. 
Table 2

Candidate (bio)markers for physical, cognitive, and psychological frailty: Results from univariate analyses.

\begin{tabular}{|c|c|c|c|c|c|c|}
\hline Domain & Biomarker or marker & $\begin{array}{l}\text { Adjusted } \\
\text { p-value }\end{array}$ & $\begin{array}{l}\text { Median } \\
\text { non-frail group }^{a}\end{array}$ & $\begin{array}{l}\text { Median } \\
\text { frail group }^{\mathrm{a}}\end{array}$ & Difference between medians ${ }^{\mathrm{b}}$ & Relative difference $(\%)^{\mathrm{c}}$ \\
\hline \multirow[t]{9}{*}{ Physical } & Self-reported health ${ }^{* \mathrm{~d}}$ & $<.0001$ & & & & \\
\hline & Excellent/ Very good/ Good & & $90 \%$ & $48 \%$ & & \\
\hline & Poor/ Fair & & $10 \%$ & $52 \%$ & & \\
\hline & $\alpha$-Carotene $(\mu \mathrm{mol} / \mathrm{l})$ & 0.0078 & 0.142 & 0.096 & -0.046 & -32.4 \\
\hline & Selenium (ppb) & 0.0098 & 109.333 & 99.495 & -9.838 & -9.0 \\
\hline & $\beta$-Cryptoxanthin $(\mu \mathrm{mol} / \mathrm{l})$ & 0.0130 & 0.211 & 0.152 & -0.059 & -28.0 \\
\hline & SeAlbSeP (ppb) & 0.0206 & 86.978 & 78.183 & -8.795 & -10.1 \\
\hline & Monocytes $\left(10^{6} / 1\right)$ & 0.0229 & 452.600 & 526.300 & 73.700 & 16.3 \\
\hline & $\beta$-Carotene $(\mu \mathrm{mol} / \mathrm{l})$ & 0.0242 & 0.565 & 0.394 & -0.171 & -30.3 \\
\hline \multirow[t]{9}{*}{ Cognitive } & Self-reported health ${ }^{* \mathrm{~d}}$ & $<.0001$ & & & & \\
\hline & Excellent/ Very good/ Good & & $92 \%$ & $73 \%$ & & \\
\hline & Poor/ Fair & & $8 \%$ & $27 \%$ & & \\
\hline & $\beta$-Cryptoxanthin $(\mu \mathrm{mol} / \mathrm{l})$ & $<.0001$ & 0.215 & 0.148 & -0.067 & -31.2 \\
\hline & Zeaxanthin $(\mu \mathrm{mol} / \mathrm{l})$ & 0.0002 & 0.044 & 0.036 & -0.008 & -18.2 \\
\hline & $\operatorname{CMV}(\mathrm{U} / \mathrm{l})^{\mathrm{f}}$ & 0.0017 & 19.300 & 36.540 & 17.240 & 89.3 \\
\hline & Cholesterol (mmol/l) & 0.0086 & 5.510 & 5.340 & -0.170 & -3.1 \\
\hline & $\alpha$-Tocopherol $(\mu \mathrm{mol} / \mathrm{l})$ & 0.0148 & 27.790 & 26.570 & -1.220 & -4.4 \\
\hline & Guanidinoacetate $3.97 \mathrm{~s}$ (area (a.u.) $)^{g}$ & 0.0480 & 8.466 & 7.616 & -0.85 & -10.0 \\
\hline \multirow[t]{9}{*}{ Psychological } & Self-reported health ${ }^{* \mathrm{~d}}$ & $<.0001$ & & & & \\
\hline & Excellent/ Very good/ Good & & $91 \%$ & $55 \%$ & & \\
\hline & Poor/ Fair & & $9 \%$ & $45 \%$ & & \\
\hline & Level of education ${ }^{* h}$ & 0.0002 & & & & \\
\hline & Low & & $10 \%$ & $25 \%$ & & \\
\hline & Middle-low & & $33 \%$ & $39 \%$ & & \\
\hline & Middle-high & & $39 \%$ & $28 \%$ & & \\
\hline & High & & $18 \%$ & $8 \%$ & & \\
\hline & $\alpha$-Carotene $(\mu \mathrm{mol} / \mathrm{l})$ & 0.0224 & 0.142 & 0.119 & -0.023 & -16.2 \\
\hline
\end{tabular}

Notes: Rank-ANOVA with adjustment for age and sex and correction for multiple testing.

"Self-reported health and level of education are binary and categorical variables, respectively. Therefore, the percentages per category of these two variables are presented and not the median as presented for the (continuous) biomarker variables.

${ }^{a}$ Medians are of untransformed biomarker levels and are not adjusted for age and sex. Participants in the non-frail group of a certain domain could be frail on another domain.

b Difference of median biomarker levels between individuals with and without frailty.

${ }^{c}$ Relative difference of median biomarker levels between individuals with and without frailty expressed in percentages.

d Self-reported health was defined as: 1 = 'fair','poor'; $0=$ 'excellent','very good','good'.

e SeAlbSeP (ppb): Plasma Selenium eluting with retention time of Albumin or Selenoprotein P (absolute value in ppb).

f CMV: cytomegalovirus antibodies.

g Relative Guanidinoacetate 3.97 s concentration.

h Level of education was divided into four categories: Low = 'Never went to school' or 'Did not finish elementary school (i.e. up to age 10)' or 'Finished elementary school'; Middle-low = 'First stage of secondary level education' or 'Second stage of secondary level education'; Middle-high = 'Recognized third level education: a third level education other than university degree' or' Recognized third level education: an initial university degree or recognized equivalent'; High = 'Recognized third level education: a higher university degree or post graduate'.

Further, we identified self-reported health as a marker for all frailty domains in the univariate analyses when adjusting for sex, and when stratifying for sex. Self-reported health, perhaps in combination with certain biomarkers, could help detect those at increased risk of frailty. Longitudinal studies are instrumental to study this further.

In the univariate analyses adjusting for sex, we found multiple biomarkers for physical and cognitive frailty, while for psychological frailty we found only one biomarker, i.e. $\alpha$-carotene. Possibly, a larger number of biological processes may be involved in physical and cognitive frailty while different factors play a role in psychological frailty. Given the overlap in types of biomarkers identified for physical and cognitive frailty, the underlying mechanisms for these domains may be related.

Frailty is recognized as a multidimensional syndrome and therefore we believe it is important to take the cognitive frailty domain into account. We deviated from the current definition for cognitive frailty (Kelaiditi et al., 2013), because this definition also includes physical components. Since it is possible to be cognitively frail without being physically frail (Fig. 1), we adjusted our operationalization accordingly.

We used two approaches, univariate and multivariate analyses, to identify (bio)markers for different frailty domains both providing relevant information (Saccenti et al., 2014). The results from the univariate analyses showed significant differences in the levels of certain (bio)markers between the frail and the non-frail groups. The advantage of this analysis is that the results are easy to interpret. The results from the multivariate analyses showed which biomarkers are associated with a certain frailty domain taking inter-variable relations into account. In our approach, we had to deal with many missing values limiting the number of (bio)markers used for the multivariate versus the univariate analyses.

The RASIG population of the MARK-AGE study spans the 'middle age range' in industrialized countries and is a relatively young population for studying (bio)markers of frailty. The low prevalence of frailty in this population made it more challenging to identify candidate (bio)markers in a multivariate approach. Furthermore, the participants for the RASIG population were recruited via newspapers, which may have introduced a selection bias. This may be reflected by the relatively high educational level of the population. Also, the biological material was obtained from participants in several European countries. This may have influenced the sample collection, storage, and measurements of 
Table 3a

Candidate (bio)markers for physical, cognitive, and psychological frailty in women: Results from univariate analyses.

\begin{tabular}{|c|c|c|c|c|c|c|}
\hline \multirow[t]{2}{*}{ Domain } & \multicolumn{6}{|l|}{ Women } \\
\hline & Biomarker or marker & $\begin{array}{l}\text { Adjusted } \\
\text { p-value }\end{array}$ & $\begin{array}{l}\text { Median } \\
\text { non-frail group }\end{array}$ & $\begin{array}{l}\text { Median } \\
\text { frail group }^{\text {a }}\end{array}$ & Difference between medians ${ }^{\mathrm{b}}$ & Relative difference (\%) ${ }^{c}$ \\
\hline \multirow[t]{5}{*}{ Physical } & Self-reported health ${ }^{* \mathrm{~d}}$ & $<.0001$ & & & & \\
\hline & Excellent/ Very good/ Good & & $89 \%$ & $47 \%$ & & \\
\hline & Poor/ Fair & & $11 \%$ & $53 \%$ & & \\
\hline & $\alpha$-Carotene $(\mu \mathrm{mol} / \mathrm{l})$ & 0.0216 & 0.172 & 0.103 & -0.069 & -40.1 \\
\hline & $\beta$-Carotene $(\mu \mathrm{mol} / \mathrm{l})$ & 0.0315 & 0.656 & 0.413 & -0.243 & -37.0 \\
\hline \multirow[t]{3}{*}{ Cognitive } & Self-reported health ${ }^{* \mathrm{~d}}$ & $<.0001$ & & & & \\
\hline & Excellent/ Very good/ Good & & $91 \%$ & $68 \%$ & & \\
\hline & Poor/ Fair & & $9 \%$ & $32 \%$ & & \\
\hline \multirow[t]{8}{*}{ Psychological } & Self-reported health ${ }^{* \mathrm{~d}}$ & $<.0001$ & & & & \\
\hline & Excellent/ Very good/ Good & & $91 \%$ & $59 \%$ & & \\
\hline & Poor/ Fair & & $9 \%$ & $41 \%$ & & \\
\hline & Level of education*e & $<.0001$ & & & & \\
\hline & Low & & $10 \%$ & $28 \%$ & & \\
\hline & Middle-low & & $32 \%$ & $40 \%$ & & \\
\hline & Middle-high & & $41 \%$ & $27 \%$ & & \\
\hline & High & & $17 \%$ & $4 \%$ & & \\
\hline
\end{tabular}

Notes: Rank-ANOVA with adjustment for age, stratification for sex, and correction for multiple testing.

"Self-reported health and level of education are binary and categorical variables, respectively. Therefore, the percentages per category of these two variables are presented and not the median as presented for the (continuous) biomarker variables.

${ }^{a}$ Medians are of untransformed biomarker levels and are not adjusted for age. Participants in the non-frail group of a certain domain could be frail on another domain.

b Difference of median biomarker levels between individuals with and without frailty.

${ }^{c}$ Relative difference of median biomarker levels between individuals with and without frailty expressed in percentages.

d Self-reported health was defined as: 1 = 'fair', 'poor'; 0 = 'excellent', 'very good', 'good'.

e Level of education was divided into four categories: Low = 'Never went to school' or 'Did not finish elementary school (i.e. up to age 10)' or 'Finished elementary school'; Middle-low = 'First stage of secondary level education' or 'Second stage of secondary level education'; Middle-high = 'Recognized third level education: a third level education other than university degree' or' Recognized third level education: an initial university degree or recognized equivalent'; High $=$ 'Recognized third level education: a higher university degree or post graduate'.

Table 3b

Candidate (bio)markers for physical, cognitive, and psychological frailty in men: Results from univariate analyses.

\begin{tabular}{|c|c|c|c|c|c|c|}
\hline \multirow[t]{2}{*}{ Domain } & \multicolumn{6}{|l|}{ Men } \\
\hline & Biomarker or marker & $\begin{array}{l}\text { Adjusted } \\
\text { p-value }\end{array}$ & $\begin{array}{l}\text { Median } \\
\text { non-frail group }\end{array}$ & $\begin{array}{l}\text { Median } \\
\text { frail group }^{\mathrm{a}}\end{array}$ & Difference between medians ${ }^{\mathrm{b}}$ & Relative difference $(\%)^{c}$ \\
\hline \multirow[t]{3}{*}{ Physical } & Self-reported health ${ }^{* \mathrm{~d}}$ & $<.0001$ & & & & \\
\hline & Excellent/ Very good/ Good & & $91 \%$ & $50 \%$ & & \\
\hline & Poor/ Fair & & $9 \%$ & $50 \%$ & & \\
\hline \multirow[t]{6}{*}{ Cognitive } & Self-reported health ${ }^{* \mathrm{~d}}$ & $<.0001$ & & & & \\
\hline & Excellent/ Very good/ Good & & $93 \%$ & $76 \%$ & & \\
\hline & Poor/ Fair & & $7 \%$ & $24 \%$ & & \\
\hline & $\beta$-Cryptoxanthin $(\mu \mathrm{mol} / \mathrm{l})$ & 0.0002 & 0.176 & 0.122 & -0.054 & -30.7 \\
\hline & $\operatorname{CMV}(\mathrm{U} /)^{\mathrm{e}}$ & 0.0004 & 14.645 & 37.180 & 22.535 & 153.9 \\
\hline & Guanidinoacetate $3.97 \mathrm{~s}(\text { area }(\text { a.u. }))^{\mathrm{f}}$ & 0.0382 & 8.180 & 7.136 & -1.044 & -12.8 \\
\hline \multirow[t]{4}{*}{ Psychological } & Self-reported health ${ }^{* d}$ & $<.0001$ & & & & \\
\hline & Excellent/ Very good/ Good & & $92 \%$ & $46 \%$ & & \\
\hline & Poor/ Fair & & $8 \%$ & $54 \%$ & & \\
\hline & CunotCp (ppb) ${ }^{g}$ & 0.0214 & 165.848 & 144.792 & -21.060 & -12.7 \\
\hline
\end{tabular}

Notes: Rank-ANOVA with adjustment for age, stratification for sex, and correction for multiple testing.

"Self-reported health and level of education are binary and categorical variables, respectively. Therefore, the percentages per category of these two variables are presented and not the median as presented for the (continuous) biomarker variables.

${ }^{a}$ Medians are of untransformed biomarker levels and are not adjusted for age. Participants in the non-frail group of a certain domain could be frail on another domain.

b Difference of median biomarker levels between individuals with and without frailty.

c Relative difference of median biomarker levels between individuals with and without frailty expressed in percentages.

d Self-reported health was defined as: 1 = 'fair', 'poor'; 0 = 'excellent', 'very good', 'good'.

e CMV: cytomegalovirus antibodies.

${ }^{\mathrm{f}}$ Relative Guanidinoacetate $3.97 \mathrm{~s}$ concentration.

g CunotCp (ppb): Plasma Copper not bound to Ceruloplasmin (absolute value in ppb). 
Table 4

Candidate (bio)markers for physical, cognitive, and psychological frailty: Results from multivariate analyses.

\begin{tabular}{|c|c|c|c|c|c|}
\hline \multicolumn{2}{|l|}{ Physical } & \multicolumn{2}{|l|}{ Cognitive } & \multicolumn{2}{|l|}{ Psychological } \\
\hline Biomarker or marker & Selection probability & Biomarker or marker & Selection probability & Biomarker or marker & Selection probability \\
\hline$\alpha$-Carotene $(\mu \mathrm{mol} / \mathrm{l})$ & 0.47 & $\beta$-Cryptoxanthin $(\mu \mathrm{mol} / \mathrm{l})$ & 0.75 & Hemoglobin (g/l) & 0.45 \\
\hline Ascorbic acid (mg/l) & 0.41 & Zeaxanthin $(\mu \mathrm{mol} / \mathrm{l})$ & 0.53 & Hematocrit (\%) & 0.30 \\
\hline Monocytes $\left(10^{6} / 1\right)$ & 0.37 & $\operatorname{CMV}(\mathrm{U} / \mathrm{l})^{\mathrm{C}}$ & 0.41 & ApoJ/Clu $(\mu \mathrm{g} / \mathrm{ml})^{g}$ & 0.28 \\
\hline$\beta$-Cryptoxanthin $(\mu \mathrm{mol} / \mathrm{l})$ & 0.32 & LDL1 Cholesterol $(\mathrm{mg} / \mathrm{dl})^{\mathrm{d}}$ & 0.36 & Tetanus IgG antibodies (IU/ml) & 0.25 \\
\hline$\beta$-Carotene $(\mu \mathrm{mol} / \mathrm{l})$ & 0.31 & PSA $(\mathrm{ng} / \mathrm{ml})^{\mathrm{e}}$ & 0.36 & 25-Hydroxy-Vitamin-D (nmol/l) & 0.25 \\
\hline Lutein $(\mu \mathrm{mol} / \mathrm{l})$ & 0.29 & HDL cholesterol $(\mathrm{mmol} / \mathrm{l})^{\mathrm{f}}$ & 0.33 & Urinary 8-isoprostane ( $\mathrm{mM} / \mathrm{l} / \mathrm{mM}$ creatinine) & 0.24 \\
\hline Insulin $(\mu \mathrm{IU} / \mathrm{ml})$ & 0.21 & $\beta$-Carotene $(\mu \mathrm{mol} / \mathrm{l})$ & 0.30 & Systolic blood pressure & 0.24 \\
\hline Research center no $2^{\mathrm{a}}$ & 0.18 & Lutein $(\mu \mathrm{mol} / \mathrm{l})$ & 0.30 & Hemoglobin $(\mathrm{g} / \mathrm{l})^{\mathrm{h}}$ & 0.23 \\
\hline$\gamma$-Tocopherol $(\mu \mathrm{mol} / \mathrm{l})$ & 0.18 & Neutrophils $\left(10^{9} / 1\right)$ & 0.28 & $\mathrm{Hb}-\mathrm{MCHC}^{\mathrm{i}}$ & 0.18 \\
\hline $\mathrm{BMI}^{\mathrm{b}}$ & 0.17 & Monocytes $\left(10^{6} / 1\right)$ & 0.24 & FHL2 CpG6 & 0.17 \\
\hline
\end{tabular}

Note: In this table, the ten biomarkers with the highest selection probability for each frailty domain are presented. Biomarkers with an average selection probability of $>0.5$ (in bold) were used for further analyses.

a Research center number 2 is BioTeSys (Germany).

b BMI: Body mass index $\left(\mathrm{kg} / \mathrm{m}^{2}\right)$.

c CMV: Cytomegalovirus antibodies.

d LDL1 Cholesterol (mg/dl): Serum concentration of cholesterol in large low-density lipoprotein particles.

e PSA: Prostate specific antigen.

${ }^{\mathrm{f}}$ HDL cholesterol: High-density lipoproteins cholesterol.

g ApoJ/Clu: Apolipoprotein J/Clusterin serum levels.

h Hemoglobin of frozen blood.

${ }^{i}$ Hb-MCHC: Mean corpuscular hemoglobin concentration.

${ }^{\mathrm{j}}$ FHL2 CpG6: DNA methylation level of a specific CpG in FHL2 gene.

Table 5

Delta for candidate biomarkers with a selection probability $>0.5$ from the multivariate analyses.

\begin{tabular}{lrr}
\hline Domain & Biomarker & Delta (90th vs 10th percentile) \\
\hline Physical & - & - \\
Cognitive & $\beta$-Cryptoxanthin & $-9.3 \%$ \\
& Zeaxanthin & $-6.3 \%$ \\
Psychological & - & -
\end{tabular}

Note: The delta, the risk of being frail, was calculated by subtracting the average value of the 10th percentile for a certain biomarker from the 90th percentile from that biomarker.

\section{Table 6}

Logistic regression analyses on biomarkers resulted from both the univariate and multivariate analyses.

\begin{tabular}{llll}
\hline Domain & Biomarker & $\begin{array}{l}\text { Model 1 OR (95\% } \\
\text { CI) }\end{array}$ & $\begin{array}{l}\text { Model 2 OR (95\% } \\
\text { CI) }\end{array}$ \\
\hline Cognitive & $\begin{array}{r}\text { Log } \beta- \\
\text { Cryptoxanthin } \\
\text { Log Zeaxanthin }\end{array}$ & $0.642(0.538-0.765)$ & $0.742(0.604-0.911)$ \\
& $0.626(0.509-0.771)$ & $0.752(0.588-0.960)$
\end{tabular}

Note: Odds ratios and $95 \%$ confidence intervals are presented in this table for biomarkers with an average selection probability of $>0.5$.

${ }^{a}$ Model 1 is a model adjusted for sex and age.

b Model 2 is a model adjusted for sex, age, level of education, BMI, smoking status, depressive symptoms and season of blood collection.

the biological material. However, due to the standardized, elaborate protocol these potential differences are assumed to be kept to a minimum. In addition, the category 'recruitment center' was included as a variable in both the univariate and multivariate analyses and did not seem to have an effect.

The potential (bio)markers for frailty we identified need to be replicated in other studies and causality could be examined in a longitudinal cohort. In particular, the latter will indicate whether antioxidants and self-reported health could serve as (bio)markers to detect people at risk of becoming frail. For preventive purposes, causality needs to be established with respect to antioxidant levels and frailty.
Underlying questions are: Is the difference caused by nutritional intake, gastrointestinal absorption, or micronutrient metabolism and does genetics play a role in (one) of these processes? Collectively, the identified biomarkers may indicate the involvement of inflammation in frailty, especially for physical and cognitive frailty.

\section{Conflict of interest}

The authors declare no conflicts of interest.

\section{Funding}

This work was supported by the Ministry of Health, Welfare and Sport of the Netherlands, the National Institute for Public Health and the Environment (grant number S132002) and the European Commission through the FP7 large-scale integrating project "European Study to Establish Biomarkers of Human Ageing" (MARK-AGE; grant agreement No.: 200880).

\section{Acknowledgements}

We would like to thank all study subjects for their participation in the MARK-AGE study and all consortium partners for their contribution to the data collection used in this study.

\section{References}

Aguayo, G.A., Donneau, A.F., Vaillant, M.T., Schritz, A., Franco, O.H., Stranges, S., et al., 2017. Agreement between 35 published frailty scores in the general population. Am. J. Epidemiol. 186 (4), 420-434.

Akbaraly, N.T., Faure, H., Gourlet, V., Favier, A., Berr, C., 2007. Plasma carotenoid levels and cognitive performance in an elderly population: results of the EVA study. J. Gerontol. Ser. A, Biol. Sci. Med. Sci. 62 (3), 308-316.

Benjamini, Y., Hochberg, Y., 1995. Controlling the false discovery rate: a practical and powerful approach to multiple testing. J. R. Statis. Soc. B 57 (1), 289-300. 
Berwick, D.M., Murphy, J.M., Goldman, P.A., Ware Jr., J.E., Barsky, A.J., Weinstein, M.C., 1991. Performance of a five-item mental health screening test. Med Care 29 (2), 169-176.

Black, C.N., Penninx, B.W., Bot, M., Odegaard, A.O., Gross, M.D., Matthews, K.A., et al., 2016. Oxidative stress, anti-oxidants and the cross-sectional and longitudinal association with depressive symptoms: results from the CARDIA study. Transl. Psychiatry 6, e743.

Blagus, R., Lusa, L., 2015. Joint use of over- and under-sampling techniques and crossvalidation for the development and assessment of prediction models. BMC Bioinform. $16(1), 363$.

Brand, N., Jolles, J., 1985. Learning and retrieval rate of words presented auditorily and visually. J. Gen. Psychol. 112 (2), 201-210.

Burkle, A., Moreno-Villanueva, M., Bernhard, J., Blasco, M., Zondag, G., Hoeijmakers, J.H., et al., 2015. MARK-AGE biomarkers of ageing. Mech. Ageing Dev. 151, 2-12.

Collerton, J., Martin-Ruiz, C., Davies, K., Hilkens, C.M., Isaacs, J., Kolenda, C., et al., 2012. Frailty and the role of inflammation, immunosenescence and cellular ageing in the very old: cross-sectional findings from the Newcastle $85+$ study. Mech. Ageing Dev. 133 (6), 456-466.

Dominguez, L.J., Barbagallo, M., 2017. The relevance of nutrition for the concept of cognitive frailty. Curr. Opin. Clin. Nutr. Metab. Care 20 (1), 61-68.

Feeney, J., O'Leary, N., Moran, R., O'Halloran, A.M., Nolan, J.M., Beatty, S., et al., 2017. Plasma lutein and zeaxanthin are associated with better cognitive function across multiple domains in a large population-based sample of older adults: findings from the Irish longitudinal study on aging. J. Gerontol. A Biol. Sci. Med. Sci. 72 (Oct. (10)), 1431-1436.

Fried, L.P., Tangen, C.M., Walston, J., Newman, A.B., Hirsch, C., Gottdiener, J., et al. 2001. Frailty in older adults: evidence for a phenotype. J. Gerontol. Series A Biol. Sci. Med. Sci. 56 (3), M146-M156.

Gobbens, R.J., van Assen, M.A., Luijkx, K.G., Wijnen-Sponselee, M.T., Schols, J.M., 2010. The Tilburg frailty indicator: psychometric properties. J. Am. Med. Directors Assoc. 11 (5), 344-355.

Hamerman, D., 1999. Toward an understanding of frailty. Ann. Intern. Med. 130 (11), 945-950.

Hubbard, R.E., O’Mahony, M.S., Savva, G.M., Calver, B.L., Woodhouse, K.W., 2009. Inflammation and frailty measures in older people. J. Cell. Mol. Med. 13 (9b), 3103-3109.

Ingles, M., Gambini, J., Mas-Bargues, C., Garcia-Garcia, F.J., Vina, J., Borras, C., 2016. Brain-derived neurotrophic factor as a marker of cognitive frailty. J. Gerontol.: Ser. A 72 (3), 450-451 1 March 2017.

Kelaiditi, E., Cesari, M., Canevelli, M., van Kan, G.A., Ousset, P.J., Gillette-Guyonnet, S., et al., 2013. Cognitive frailty: rational and definition from an (I.A.N.A./I.A.G.G.) international consensus group. J. Nutr. Health Aging 17 (9), 726-734.

Leng, S.X., Xue, Q.L., Tian, J., Walston, J.D., Fried, L.P., 2007. Inflammation and frailty in older women. J. Am. Geriatr. Soc. 55 (6), 864-871.

Lezak, M.D., Howieson, D.B., Loring, D.W., 2004. In: Stroop, J.R. (Ed.), Neuropsychological Assessment.Studies of Interference in Serial Verbal Reactions. OxfordUniversity Press, New York, pp. 368-370.

Maggio, M., Guralnik, J.M., Longo, D.L., Ferrucci, L., 2006. Interleukin-6 in aging and chronic disease: a magnificent pathway. J. Gerontol. Ser. A. 61 (6), 575-584.

Maggio, M., De Vita, F., Lauretani, F., Butto, V., Bondi, G., Cattabiani, C., et al., 2013. IGF1 , the cross road of the nutritional, inflammatory and hormonal pathways to frailty. Nutrients 5 (10), 4184-4205.

Meinshausen, N., Bühlmann, P., 2010. Stability selection. J. R. Statis. Soc. 72 (4), $417-473$.

Michelon, E., Blaum, C., Semba, R.D., Xue, Q.L., Ricks, M.O., Fried, L.P., 2006. Vitamin and carotenoid status in older women: associations with the frailty syndrome. The journals of gerontology series A. Biol. Sci. Med. Sci. 61 (6), 600-607.

Mitnitski, A., Collerton, J., Martin-Ruiz, C., Jagger, C., von Zglinicki, T., Rockwood, K., et al., 2015. Age-related frailty and its association with biological markers of ageing. BMC Med. 13, 161.

Moreno-Villanueva, M., Capri, M., Breusing, N., Siepelmeyer, A., Sevini, F., Ghezzo, A., et al., 2015a. MARK-AGE standard operating procedures (SOPs): A successful effort.
Mech. Ageing Dev. 151, 18-25.

Moreno-Villanueva, M., Kotter, T., Sindlinger, T, Baur, J, Oehlke, S., Burkle, A, et al, 2015b. The MARK-AGE phenotypic database: Structure and strategy. Mech. Ageing Dev. 151, 26-30.

Morley, J.E., Baumgartner, R.N., 2004. Cytokine-related aging process. J. Gerontol. Ser. A Biol. Sci. Med. Sci. 59 (9), M924-M929.

Peltonen, L., Jalanko, A., Varilo, T., 1999. Molecular genetics the Finnish disease heritage. Hum. Mol. Genet. 8 (10), 1913-1923.

Perenboom, R.O.K., van Herten, L., Hoeymans, N., Bijl, R., 2000. Life-expectancy in Good Mental Health: Establishing Cut-Off Points for the MHI-5 and GHQ-12. in Dutch. Leiden: TNO-report PG/VGZ/99067.

Rietman, M.L., van der, A.D.L., van Oostrom, S.H., Picavet, H.S.J., Dolle, M.E.T., van Steeg, H., et al., 2018. The association between BMI and different frailty domains: a U-shaped curve? J. Nutr. Health Aging 22 (1), 8-15.

Rockwood, K., Stadnyk, K., MacKnight, C., McDowell, I., Hebert, R., Hogan, D.B., 1999. A brief clinical instrument to classify frailty in elderly people. Lancet (London, England). 353 (9148), 205-206.

Saccenti, E., Hoefsloot, H.C.J., Smilde, A.K., Westerhuis, J.A., Hendriks, M.M.W.B., 2014. Reflections on univariate and multivariate analysis of metabolomics data. Metabolomics 10 (3), 361-374.

Saum, K.U., Dieffenbach, A.K., Jansen, E.H., Schottker, B., Holleczek, B., Hauer, K., et al., 2015. Association between oxidative stress and frailty in an elderly German population: results from the ESTHER cohort study. Gerontology 61 (5), 407-415.

Semba, R.D., Lauretani, F., Ferrucci, L., 2007. Carotenoids as protection against sarcopenia in older adults. Arch. Biochem. Biophys. 458 (2), 141-145.

Smit, E., Winters-Stone, K.M., Loprinzi, P.D., Tang, A.M., Crespo, C.J., 2013. Lower nutritional status and higher food insufficiency in frail older US adults. Br. J. Nutr. 110 (1), 172-178.

Speechley, M., Tinetti, M., 1991. Falls and injuries in frail and vigorous community elderly persons. J. Am. Geriatr. Soc. 39 (1), 46-52.

Stroop, J.R., 1935. Studies of interference in serial verbal reactions. J. Exp. Psychol. 18, 643-662.

Stuetz, W., Weber, D., Dolle, M.E., Jansen, E., Grubeck-Loebenstein, B., Fiegl, S., et al., 2016. Plasma carotenoids, tocopherols, and retinol in the age-stratified (35-74 years) general population: a cross-sectional study in six European countries. Nutrients 8 (10).

Valko, M., Rhodes, C.J., Moncol, J., Izakovic, M., Mazur, M., 2006. Free radicals, metals and antioxidants in oxidative stress-induced cancer. Chem.-Biol. Interact. 160 (1), $1-40$.

Vina, J, Tarazona-Santabalbina, F.J., Perez-Ros, P., Martinez-Arnau, F.M., Borras, C. Olaso-Gonzalez, G., et al., 2016. Biology of frailty: modulation of ageing genes and its importance to prevent age-associated loss of function. Mol. Aspects Med. 50, 88-108.

Class imbalance, redux. In: Wallace, B.C., Small, K., Brodley, C.E., Trikalinos, T.A. (Eds.), 2011 IEEE 11th International Conference on Data Mining, 11-14 Dec. 2011.

Walston, J., McBurnie, M.A., Newman, A., Tracy, R.P., Kop, W.J., Hirsch, C.H., et al., 2002. Frailty and activation of the inflammation and coagulation systems with and without clinical comorbidities: results from the cardiovascular health study. Arch. Intern. Med. 162 (20), 2333-2341.

Walston, J., Hadley, E.C., Ferrucci, L., Guralnik, J.M., Newman, A.B., Studenski, S.A., et al., 2006. Research agenda for frailty in older adults: toward a better understanding of physiology and etiology: summary from the American geriatrics society/ national institute on aging research conference on frailty in older adults. J. Am. Geriatr. Soc. 54 (6), 991-1001.

Winograd, C.H., Gerety, M.B., Chung, M., Goldstein, M.K., Dominguez Jr., F., Vallone, R., 1991. Screening for frailty: criteria and predictors of outcomes. J. Am. Geriatr. Soc. 39 (8), 778-784.

Yeo, I.K., Johnson, R.A., 2000. A new family of power transformations to improve normality or symmetry. Biometrika 87 (4), 954-959.

Zou, H., Hastie, T., 2005. Regularization and variable selection via the elastic net. J. R. Statis. Soc. 67 (2), 301-320.

Zung, W.W., 1965. A SELF-RATING DEPRESSION SCALE. Arch. Gen. Psychiatry 12, 63-70. 